\title{
Evaluation of TRMM Ground-Validation Radar-Rain Errors Using Rain Gauge Measurements
}

\author{
JANXIN WANG ${ }^{1,2}$ AND DAVID B. WOLFF ${ }^{1,2}$ \\ ${ }^{1}$ Science System and Applications, Inc., Lanham, Maryland 20706 \\ ${ }^{2}$ NASA Goddard Space Flight Center, Greenbelt, Maryland 20706
}

\begin{abstract}
Submitted
Journal of Applied Meteorology and Climatology
\end{abstract}

April 2009

Corresponding author address: Jianxin Wang, NASA Goddard Space Flight Center, Code 613.1, Greenbelt, MD 20771.

E-mail: jianxin.wang@nasa.gov 


\begin{abstract}
Ground-validation (GV) radar-rain products are often utilized for validation of the Tropical Rainfall Measuring Mission (TRMM) spaced-based rain estimates, and hence, quantitative evaluation of the GV radar-rain product error characteristics is vital. This study uses quality-controlled gauge data to compare with TRMM GV radar rain rates in an effort to provide such error characteristics. The results show that significant differences of concurrent radar-gauge rain rates exist at various time scales ranging from $5 \mathrm{~min}$ to 1 day, despite lower overall long-term bias. However, the differences between the radar area-averaged rain rates and gauge point rain rates cannot be explained as due to radar error only. The error variance separation method is adapted to partition the variance of radar-gauge differences into the gauge area-point error variance and radar rain estimation error variance. The results provide relatively reliable quantitative uncertainty evaluation of TRMM GV radar rain estimates at various times scales, and are helpful to better understand the differences between measured radar and gauge rain rates. It is envisaged that this study will contribute to better utilization of GV radar rain products to validate versatile spaced-based rain estimates from TRMM, as well as the proposed Global Precipitation Measurement, and other satellites.
\end{abstract}




\section{Introduction}

The Tropical Rainfall Measuring Mission (TRMM) is a satellite-based program to estimate global tropical rainfall (Simpson et al. 1996; Kummerow et al. 1998). A wealth of data has been obtained since the launch of the TRMM satellite in November 1997. The accuracy of the satellite-based rain products, however, is affected by discrete temporal sampling and remote spaceborne rain retrieval algorithms. The TRMM ground validation (GV) program was established to verify the satellite rain products, which can be carried out by comparing the TRMM products with ground observations from ground sensors, such as rain gauges, radars and disdrometers. The GV radar-rain products play a crucial role in this validation effort. TRMM Standard Product (TSP) 2A-53, an instantaneous GV radar rain-rate map, is often used as a main tool for the validation analysis (Adler et al. 2000; Habib and Krajewski 2002; Datta et al. 2003; Wolff et al. 2005). However, TSP $2 \mathrm{~A}-53$ has its own quality issues which must be quantified before it can be used to properly validate the satellite rain estimates. The major obstacle in evaluation of the quality of the $2 \mathrm{~A}-53$ is the lack of "ground truth" reference at the radar pixel scale $(2 \mathrm{~km} \times 2 \mathrm{~km})$. The surface rainfall measurements from rain gauges are customarily approximated as the "ground truth" to assess and calibrate radar-rain estimates.

The rain gauge is a simple mechanical device that is deployed on the surface to directly measure rainwater entering the gauge in discrete quantities. For example, Met One ${ }^{\circledR}$, a commonly used tipping-bucket (TB) rain gauge, measures rainwater impinging on its 30.5 -cm-diameter collection orifice and entering into its $0.254 \mathrm{~mm}$ bucket. Subsequently, the TB gauge gives the surface rainfall amounts in a near-point area of 
$0.073 \mathrm{~m}^{2}$ with the sampling resolution of $0.254 \mathrm{~mm}$. The radar, in contrast, does not directly measure surface rainfall amounts, but measures reflectivity factors aloft at its resolution cell in the order of 1 to $10 \mathrm{~km}^{2}$. The area-averaged surface rainfall is subsequently estimated via converting raw reflectivity using any number of reflectivity rain-rate $\left(Z_{\mathrm{e}}-\mathrm{R}\right)$ relationships. Even if both gauge near-point measurements and radar area-averaged rainfall estimates are assumed to be accurate, the large resolution difference of 7-8 orders of magnitude still obviously causes problematic comparisons between two sensors. Because gauges lack areal representativeness, the gauge rain measurements cannot be directly treated as the "ground truth" reference for the areaaveraged rainfall. Therefore, the large radar-gauge discrepancies cannot be treated as radar-rain estimation error (Zawadzki 1973; Harrold et al. 1974; Krajewski 1975; Kitchen and Blackall 1992; Ciach and Krajewski 1999; Anagnostou and Krajewski 1999; Habib and Krajewski 2002; Habib et al. 2004).

For the purpose of decomposing radar-gauge difference variance into the radarrain estimation error variance and the gauge representativeness error variance, the general concept of the statistical error variance separation was proposed by Barnston (1991) and Kitchen and Blackall (1992). Barnston (1991) estimated the bias of radar and gauge area average rainfall measurements over relatively large domain sizes in the order of $100 \mathrm{~km}^{2}$. Kitchen and Blackall (1992) investigated the gauge representativeness problem in gaugeradar comparisons at radar pixel scale. Ciach and Krajewski (1999) fully formulated the concept as the error variance separation method (EVSM). Following the same concept, Anagnostou and Krajewski (1999) proposed a similar methodology but applied it in the logarithmic domain. Habib and Krajewski (2002) applied the ESVM formulated by Ciach 
and Krajewski (1999) to the uncertainty analysis of TRMM GV radar-rain maps for central Florida. Fisher (2007) used the ESVM to establish an upper bound of ground data uncertainty in the error decomposition of regional-scale monthly precipitation estimates from TRMM satellite. These studies showed that significant random errors existed between the rainfall estimates from the radars and gauges and that the errors decreased as the integration time scale increased.

The objective of this study is to evaluate the TRMM 2A-53 radar-rain product using rain gauge measurements. The ground rainfall data collected from a gauge network deployed in Kennedy Space Center (KSC) are compared with the concurrent overlapping radar-rain estimates. We begin with a discussion of the gauge and radar data in section 2 . Section 3 provides comparisons of gauge and radar rain-rate estimates. Application of the EVSM to quantify the contributions of gauge and radar error variances to the total radargauge difference variance is provided in section 4 . Finally a discussion of the results is given in section 5.

\section{Gauge and radar rain products}

This study uses the radar rain product $2 \mathrm{~A}-53$ and gauge rain product $2 \mathrm{~A}-56$ over KSC area in the period from January 1 to December 31 2008. Figure 1 shows a map of the KSC gauge network along with the $2 \mathrm{~km} \times 2 \mathrm{~km}$ Cartesian grids of the 2A-53 radarrain map. The dots denote locations of the individual gauges with their identification numbers indicated on the right. As can be seen in Fig. 1, the gauges are approximately evenly distributed across the study area. The distances between the gauges range from about 2 to $32 \mathrm{~km}$. The KMLB radar, a Weather Surveillance Radar -1988 Doppler 
(WSR-88D), located at $28.1133^{\circ} \mathrm{N}$ latitude and $80.6542^{\circ} \mathrm{W}$ longitude in Melbourne, Florida, is approximately $50 \mathrm{~km}$ south of the center of the KSC gauge network. The gauges are TB type with bucket size of $0.254 \mathrm{~mm}$, and are connected to an automatic data collection system. There are 31 active TB gauges regularly reporting rain tips at sampling resolution of $1 \mathrm{sec}$.

Data from a given TB gauge are subject to a number of possible error sources such as inadequate calibration before and after deployment, and mechanical and electrical problems due to the harsh environment of coastal Florida. In addition, gauge data error sources could result from the sampling mechanism, wind effects, off-level gauge placement, funnel surface wetting and evaporation, or animal and human interference, etc. Obtaining reliable measurements of point-rainfall from gauges poses difficult challenges (Sieck et al 2007). In this study, unreliable gauge tip records are detected and removed through careful quality control of the gauge data. These quality-controlled tip records are then interpolated into 1-min rain rates, known as TSP 2A-56, via a cubic spline-based algorithm described in Wang et al. 2008. Additional quality control is performed to filter "bad gauges" on a monthly basis by comparing $2 \mathrm{~A}-56$ rain rates with radar reflectivity data over the locations of rain gauges, as described by Amitai 2000. Only reliable $2 \mathrm{~A}-56$ rain rates from $\mathrm{KSC}$ gauges that passed these quality control steps are used in this study to compare with the rain rates from radar- rain product $2 \mathrm{~A}-53$.

The radar-rain product evaluated in this study is TSP $2 \mathrm{~A}-53$, an instantaneous radar rain-rate map in Cartesian coordinates with a $2-\mathrm{km}$ horizontal resolution at the $1.5-$ km Constant Altitude Plan Position Indicator (CAPPI) level. The radar rain rates are generated using reflectivity - rain-rate $\left(\mathrm{Z}_{\mathrm{e}}-\mathrm{R}\right)$ relations derived from the Window 
Probability Matching Method (WPMM; Rosenfeld et al. 1994). The WPMM statistically matches quality-controlled reflectivities extracted from radar volume scans to gaugeestimated rain rates (2A-56), such that the monthly probability distribution of the radar rain rates above the gauge is equal to that of the gauge rain rates. The derived $2 \mathrm{~A}-53$ instantaneous rain-rate maps are available approximately every 5 minutes corresponding to the volume scan interval of the KMLB radar (Wolff et al. 2005). Rain accumulations at 5-min or larger time scales are obtained by integrating the $2 \mathrm{~A}-53$ maps over the time difference between consecutive radar scans. If the time difference exceeds 12 minutes, the $2 \mathrm{~A}-53$ maps are integrated forward for 5 minutes.

It should be noted that the total of 214 gauges within $150-\mathrm{km}$ range to the KMLB radar from three separate networks are used in the WPMM. Besides 31 gauges from the KSC network, 125 gauges are from the South Florida Water Management District network (SFL) and 58 gauges are from the St. Johns Water Management District network (STJ). The percentage of the KSC gauges among total gauges used in the 2A-53 generation is $14.5 \%$. Although the $\mathrm{KSC}$ gauge data are not independent to the $2 \mathrm{~A}-53$ rain rates, they can be used in partially dependent algorithm and technique sanity checks for the long-term radar rain estimation. As the uncertainty of radar rain estimates mainly results from the radar area-averaged and gauge near-point measurements, dependent gauges can be also used in the uncertainty evaluation of radar rain estimates (Ciach and Krajewski 1999).

The gaps in the series of radar scans can cause negative bias in radar rain integration. During data processing, a gap is defined as the time difference between any two consecutive scans greater than $12 \mathrm{~min}$. This gap definition is chosen because 
consecutive scans quite often occur 11-12 min apart when the KMLB radar operates in clear-air mode. Table 1 lists monthly KMLB radar gap statistics and missing rain percentages due to the gaps in 2008 . The missing rain percentages are estimated from the percentages of gauge rainfall during the radar gaps. The monthly total radar gap length varies from $208 \mathrm{~min}$ to $7144 \mathrm{~min}$, with the largest gap percentage (16\%) reported in July when the KMLB radar experienced an antenna problem. There are a total 416 gaps comprising a total length of 23,292 minutes during the entire period of 2008 , which accounts for $4.42 \%$ of the period. The individual gap length ranges from 13 to $1945 \mathrm{~min}$ with the mean of $55.99 \mathrm{~min}$. The gaps falling in rainy periods result in about $3.49 \%$ underestimation in yearly rainfall accumulation. This percentage reduces to $0.74 \%$ if July is excluded from the calculation. About $35.97 \%$ of monthly rain accumulation in July was missed due to the radar data gaps that were often within the rainy periods. The radar gaps during dry periods do not result in missing rain such as in February and December as indicated in Table 1. In general, the radar data gap is not a serious problem in regard to the overall uncertainty of radar rain estimation. Nevertheless, all radar gaps are excluded in our comparisons of radar and gauge rainfall, as well as the error variance analysis.

\section{Comparisons of radar and gauge rainfall}

a. Overall bias

In order to evaluate the overall radar rainfall error, we define the overall bias as the ratio of the radar-gauge rainfall difference to gauge rainfall: 


$$
\operatorname{bias}=\frac{\sum_{i=1}^{N}\left(R_{i}-G_{i}\right)}{\sum_{i=1}^{N} G_{i}}
$$

where $G_{i}$ is the cumulative rainfall from a gauge over a period of a month or year, $R_{i}$ is the cumulative rainfall from a radar $2 \mathrm{~km} \times 2 \mathrm{~km}$ grid where a gauge is located in the same period, and $N$ is the number of quality-controlled gauges in the KSC network. $N$ varies from month to month. Only grids containing gauges that passed the quality control are used in the calculation of Eq. (1). Gauge data during radar data gap periods are not included in the computation of the cumulative rainfall.

Figure 2 shows the cumulative rainfall averaged from all gauges $\left(\frac{1}{N} \sum_{i=1}^{N} G_{i}\right)$ and radar grids $\left(\frac{1}{N} \sum_{i=1}^{N} R_{i}\right)$ over the gauges during the 1-year period of 2008. An average KSC gauge measured $1560.0 \mathrm{~mm}$ of rainfall while the KMLB radar estimated $1474.4 \mathrm{~mm}$ of rainfall over the same domain in 2008 . The radar overall bias relative to gauges, as defined in (1), is $-5.48 \%$. The correlation coefficient between the daily rainfall from the radar and gauge is 0.97 . Table 2 lists monthly KMLB radar and KSC gauge rainfall, as well as the radar rainfall bias relative to gauge rainfall. The monthly gauge rainfall ranges from $15.5 \mathrm{~mm}$ in December to $678.4 \mathrm{~mm}$ in August. The radar rainfall displays similar features. Note that gauge data during the radar gaps are excluded from the computation of the rain accumulation. Consequently, both radar and gauge yearly rain accumulations in Table 2 and Fig. 2 are underestimated by about $3.49 \%$ because of the radar gaps as suggested in Table 1. It should be mentioned that the KSC gauges were used in the WPMM, and therefore, the overall bias could be larger if independent gauges were 
available for this analysis. Nevertheless, the overall quantitative agreement between the KSC gauge and KMLB radar rain rates is good, considering the much larger bias of the radar rain maps due to radar calibration, estimation of the low-altitude vertical profile of reflectivity, and the $Z_{\mathrm{e}}-\mathrm{R}$ relationship (Houze et al. 2004; Wang and Wolff 2009). This confirms that the overall long-term bias is an insignificant source of uncertainties in the radar rain maps (Kitchen and Blackall 1992). The overall long-term bias of radar-rain products can be easily removed by adjusting the radar rain total to the gauge rain total. This needs to be done prior to the EVSM application.

\section{b. Scatter comparisons}

Detailed comparisons between the gauge and radar rain rates can be performed based on pairs of concurrent radar-gauge observations via scatter plots. Since TSP 2A-53 radar rain maps are instantaneous estimates sampled at about 5-min interval, we integrate radar rain maps to 6 time scales: $5,10,15,30,60$ min and 1 day, respectively. Similarly, gauge rain rates are also accumulated to these scales. Scatter plots in Fig. 3 are constructed using pairs of rain rates from all gauges and their corresponding radar grids at 6 time scales over the period of 2008. The data pairs are plotted only when both radar and gauge reported rainfall so that data gaps and large amounts of non-rainy periods are excluded from the comparisons. Both radar and gauge rain rates are plotted in the same logarithmic scale for all time scales for easy comparisons. The vertical binning of gauge rain rates can be seen in Fig. 3. This is due to the fact that the TB gauge always records rain amount in increments of $0.254 \mathrm{~mm}$ (Wang et al. 2008). The radar rain rates appear to 
be binned as evident by the horizontal lines, which is due to the precision of $0.1 \mathrm{~mm} \mathrm{~h}^{-1}$ in the $2 \mathrm{~A}-53$.

At shorter time scales of 5-30 min, significant scatter exists at both high and low rain rates, and the difference between radar and gauge rates can be beyond $\pm 100 \mathrm{~mm} \mathrm{~h}^{-1}$. The discrepancy is mainly due to different temporal and spatial samplings of the two sensors. The KMLB radar scans at about 5-min interval over a much larger area; as a result, it may miss or average out rain peaks. A gauge samples rainfall in a near-point area relative to the radar pixel. Given the large spatial-temporal variability of subtropical rainfall, the gauge may record an amount of rainfall significantly different from the pixelaveraged radar rainfall. The scatter decreases as the time scale increases from $5 \mathrm{~min}$ to 1 day. At the 60-min and 1-day scales, the scatter is relatively smaller in comparison with 5-15 min scales. Correspondingly, the correlation coefficient between the gauge and radar rain rates increases from 0.60 at 5 -min scale to 0.93 at 1 -day scale.

\section{c. Frequency distribution comparisons}

The scatter plots in Fig. 3 provide direct comparisons of GV radar and gauge rain rates, which are admittedly rather noisy. An alternative method of comparison is to analyze the frequency distributions of the differences (R-G) of the radar-gauge rain rates at various time scales (Fig. 4). Each distribution in Fig. 4 is constructed using all rainy radar-gauge data pairs covering the 1-year study period and is binned at $0.5 \mathrm{~mm} \mathrm{~h}^{-1}$ intervals. All of these distributions appear relatively symmetrical with respect to their modes and have shapes of approximate Gaussian distribution with means ranging from 1.4 to $-0.1 \mathrm{~mm} \mathrm{~h}^{-1}$ and standard deviations from 12.9 to $0.5 \mathrm{~mm} \mathrm{~h}^{-1}$. Note that the much 
larger frequency scale is used in plotting the bottom right panel, and ranges of rain-rate differences are plotted from -40 to $40 \mathrm{~mm} \mathrm{~h}^{-1}$ for clear exhibition of general distributions. The radar-gauge rain-rate differences at 5-min scale are distributed with the widest range from -196.4 to $156.7 \mathrm{~mm} \mathrm{~h}^{-1}$. The differences at 1-day scale are distributed at a narrower range than those at other scales. The range decreases with the increasing time scale from 5 min to 1 day, as indicated by the standard deviation in each panel. The coefficient of variation, defined as the ratio of the standard deviation to the mean, changes from -9.2 to -5.0 , indicating significant difference between concurrent radar and gauge rain rates, especially at short time scales. This feature is consistent with the scatter plots in Fig. 3. Although the mode of each frequency distribution is approximately zero, extreme differences do exist especially at 5- and 10-min time scales, a predictable result of the different temporal and spatial samplings of the radar and gauges as discussed earlier.

\section{d. Gauge and radar zero-rainfall percentages}

A rain gauge measures rainfall falling into its near-point circular orifice at discrete time intervals whereas the radar estimates rainfall at a much larger spatial resolution during its scans. Rain often occurs with intermittences in space and time. A few nonrainy areas may be embedded in a larger rainy area of a radar grid size; rain stoppages may exist in a heavy rain event. Hence, it is frequently observed that radar reports rain activity whereas gauges record zero rainfall. The opposite situation sometimes happens in short-lived or fast-moved storms due to discrete radar volume scans. The horizontal rain advection and vertical echo variation from radar observation height to the surface can also result in the radar missing rain. Figures $5 \mathrm{a}, \mathrm{b}$ show the percentage of gauge zero- 
rainfall occurrences conditional on radar rainfall existence in the $2 \mathrm{~km} \times 2 \mathrm{~km}$ radar grid over the gauge, and the percentage of radar zero-rainfall occurrences when the gauge inside the radar grid recorded nonzero rainfall. A general feature of Figs. 5a, b is that both percentages of conditional gauge and radar zero-rainfall occurrences are lower at larger time scales. At the radar scan frequency or the time scale of 5-min, there is about $44 \%$ chance that a single gauge does not record rainfall falling into a $2 \mathrm{~km} \mathrm{x} 2 \mathrm{~km}$ area. This percentage of conditional gauge zero-rainfall occurrence drops slowly from $44 \%$ to $36 \%$ when the time scale increases from 5-min to 1-day, whereas the percentage of conditional radar zero-rainfall occurrences drops much faster.

Similar studies about the probability of conditional zero-rainfall occurrences can be found in Habib and Krajewski (2002) and Villarini et al. (2008) where both analyses were based on gauges only, and the probability was defined as the percentage of zero-rain occurrences at one gauge conditional on rain existence at any other gauges within a given area. Compared to our analysis, Habib and Krajewski (2002) reported a relatively conservative result whereas Villarini et al. (2008) observed a higher probability. The differences are mainly due to different gauge densities, grid sizes and rainfall regimes in these three studies.

Analogous to the percentage of conditional zero-rain occurrences, the percentage of gauge missing-rainfall amount can be approximated as the ratio of radar rainfall conditional on gauge zero-rainfall in the radar grid over the gauge to the total radar rainfall, and the percentage of radar missing-rainfall amount can be defined as the ratio of gauge-rainfall conditional on radar zero-rainfall to the total gauge rainfall. The percentage of gauge missing-rainfall is about $30 \%$ at 5 -min scale, and drops to about $25 \%$ 
at 1-day scale (Fig. 5c). In comparison with the gauge missing-rainfall, the radar missingrainfall is in much smaller percentages (Fig. 5d). In general, these percentages in Figs. 5a-d indicate the importance and difficulty of radar rainfall evaluation using gauge rainfall measurements.

\section{Error variance separation method}

\section{a. Decomposition of radar-gauge difference variance}

From the analysis in section 3 , significant differences of concurrent radar-gauge rain rates exist mainly because the KMLB radar and KSC gauges sample extremely variable rainfall at different spatial and temporal resolutions. Given the huge resolution difference of 7-8 orders of magnitude in area, poor direct comparisons of radar and gauge rain rates can be expected. Thus it is fundamentally difficult to directly use gauge pointmeasurements as the "ground truth" for the radar area-averaged rainfall estimates. Many researchers have realized that the gauge-radar difference cannot be treated as radarrainfall estimation error (e.g., Zawadzki 1973; Krajewski 1987; Kitchen and Blackall 1992; Habib and Krajewski 2002). A systematic statistical approach was developed by Ciach and Krajewski (1999) to filter out the uncertainty of gauge measurements that resulted from lack of area representativeness. The basic idea of the EVSM is to separate the radar-gauge difference variance into two terms: gauge representativeness error variance and radar rainfall estimation error variance.

In a given integration time interval, similar to Ciach and Krajewski (1999) and Habib and Krajewski (2002), the variance of radar-gauge rain difference can be described as follows: 


$$
\begin{aligned}
& \operatorname{var}\left(R_{r}-R_{g}\right)=\operatorname{var}\left[\left(R_{r}-R_{t}\right)-\left(R_{g}-R_{t}\right)\right] \\
& =\operatorname{var}\left(R_{r}-R_{t}\right)-2 \operatorname{cov}\left[\left(R_{r}-R_{t}\right),\left(R_{g}-R_{t}\right)\right]+\operatorname{var}\left(R_{g}-R_{t}\right)
\end{aligned}
$$

Here $R_{r}$ is the $2 \mathrm{~A}-53$ rain rate over a given radar grid; $R_{g}$ is the $2 \mathrm{~A}-56$ rain rate from a gauge located in the radar grid; and $R_{t}$ is the unknown true area-averaged rain rate over the same grid. For an integration time interval $T$ and a radar grid domain $A, R_{t}$ is defined as

$$
R_{t}=\frac{1}{T} \int_{0}^{T}\left[\frac{1}{A} \iint_{A} R(x, y, t) d x d y\right] d t
$$

where $R(x, y, t)$ is the unknown true instantaneous rain rate at aiven time $t$ and a given point with Cartesian coordinates $(x, y)$ in the continuous radar grid domain $A$. The terms $\operatorname{var}\left(R_{r}-R_{t}\right)$ and $\operatorname{var}\left(R_{g}-R_{t}\right)$ in Eq. (2) are the variance of radar-rain estimation error and variance of gauge representativeness error, with respect to the true area-averaged rain rate $R_{t}$. If the radar and gauge errors are assumed to be uncorrelated (Ciach and Krajewski 1999), the covariance term $\operatorname{cov}\left(R_{r}-R_{t}, R_{g}-R_{t}\right)$ becomes 0 , and Eq. (2) can be simplified as

$$
\operatorname{var}\left(R_{r}-R_{g}\right)=\operatorname{var}\left(R_{r}-R_{t}\right)+\operatorname{var}\left(R_{g}-R_{t}\right)
$$

Equation (4) means that the radar-gauge differences are attributed by not only radar estimation error but also gauge representativeness error. By reforming (4), the variance of radar-rain estimation error can be written as

$$
\operatorname{var}\left(R_{r}-R_{t}\right)=\operatorname{var}\left(R_{r}-R_{g}\right)-\operatorname{var}\left(R_{g}-R_{t}\right)
$$

Based on Eq. (5), we can assess the variance of radar-rain estimation error by subtracting the variance of gauge representativeness error from the variance of radar-gauge differences. The variance of radar-gauge differences in (5) can be easily computed from $n$ pairs of gauge and radar rain rates using following formula: 


$$
\operatorname{var}\left(R_{r}-R_{g}\right)=\frac{1}{n} \sum_{i=1}^{n}\left[R_{r}(i)-R_{g}(i)\right]^{2}
$$

Equation (6) is derived after the radar long-term bias is removed, which can be carried out by adjusting radar-rain integration to the gauge rain accumulation over the entire data period. After removing the radar long-term bias and assuming that the long-term gauge accumulation is free of any errors, the true area-averaged mean rain rate $m$ is the same as the gauge or radar mean rain rate:

$$
m=\frac{1}{n} \sum_{i=1}^{n} R_{r}(i)=\frac{1}{n} \sum_{i=1}^{n} R_{g}(i)=\frac{1}{n} \sum_{i=1}^{n} R_{t}(i) .
$$

The second term $\operatorname{var}\left(R_{g}-R_{t}\right)$ at right hand side of Eq.(5), often referred to as the areapoint variance, needs to be carefully addressed.

Assuming second order homogeneity of rainfall within the domain $A$, and following Ciach and Krajewski (1999), the area-point variance can be expressed in terms of the correlation function:

$$
\operatorname{var}\left(R_{g}-R_{t}\right)=\sigma_{g}^{2}\left\{1-\frac{2}{A} \iint_{A} r\left(x, y, x_{g}, y_{g}\right) d x d y+\frac{1}{A^{2}} \iint_{A}\left[\iint_{A} r(x, y) d x d y\right] d x d y\right\},
$$

where $\sigma_{g}$ is the variance of gauge rain rates, $r$ is the spatial correlation function, $x$ and $y$ are the same as in Eq. (3), $x_{g}$ and $y_{g}$ denotes the gauge location in the radar grid. According to (8), estimation of the area-point variance requires a detailed rainfall correction structure at small scales.

\section{b. Correlation structure}

This section provides the analysis of small-scale correlation structure for the purpose of estimating the area-point variance using Eq. (8). The correlation coefficient is calculated for each pair of time series of gauge rain rates at a given time scale using 
standard Pearson product-moment formula. Figure 6 displays the scatter of correlation coefficients versus distances among gauges at accumulation time scales ranging from 1 min through 1 day. Each point represents a correlation coefficient for a pair of gauges separated by a certain distance. As expected, Fig. 6 displays a clear feature that the overall correlation level increases with the time scale, and decreases with the gauge separation distance.

Although the correlation coefficient is commonly used to characterize the complicated rainfall spatial structure, accuracy and reliability associated with its estimation are not completely resolved (Stedinger 1981; Shimizu 1993; Lai et al. 1999; Ciach and Krajewski 2006). The correlation using traditional Pearson formula can be overestimated in some cases for which rain rates follow non-normal distributions (Kowalski 1972). Stedinger (1981) showed that unbiased correlations could be estimated using logarithm-transformed data. Shimizu (1993) and Habib et al. (2001a) further studied this issue, and proposed similar transformation-based procedures to better estimate the correlations. However, Ciach and Krajewski (2006) and Villarini et al. (2008) investigated the distribution of rain rates, and found that the transformation-based procedure proposed by Habib et al. (2001a) was not suitable for their studies. We performed the logarithmical transformation to the radar and gauge data, and then estimated the correlation coefficient using transformed data. The result shows the correlation slightly increases after the transformation. In comparison with the transformation-based estimation, we believe the traditional Pearson formula does not overestimate the true correlation in this study. Therefore, we directly use the traditional Pearson formula in the correlation analysis. 
The following two-parameter exponential model with the so-called "nugget" effect is chosen to approximate the spatial correlation function $r$ over separation distance $d$ between two gauges:

$$
r(d)=r_{0} \exp \left(-\frac{d}{d_{0}}\right)
$$

In Eq. (9), parameter $r_{0}$ is the immediate correlation jump or nugget according to the geostatistical literature (Cressie 1993), and (1-ro) is called the local decorrelation that represents the field correlation drop at small distances. The decorrelation can be caused by the small-scale variability of rainfall or random errors. Parameter $d_{0}$ is the correlation distance that characterizes the correlation decay. The nugget effect model is often applied in statistical correlation to analyze small-scale variability (Ciach and Krajewski 1999, 2006; Habib and Krajewski 2002; Krajewski et al. 2003).

Different procedures can be considered to estimate parameters $r_{0}$ and $d_{0}$. Ciach and Krajewski (1999) adopted a simple best-fit to the correlation structure that is presented by Krajewski and Duffy (1988) in a simulation study. A much larger correlation distance $d_{0}$ relative to the radar grid size was obtained, thus the exponential part in Eq. (9) was close to 1 and the correlation structure was dominated by the correlation jump $r_{0}$. Based on the assumption of the second order homogeneity, $r_{0}$ was estimated using the method of moments. However, this procedure over-simplified the correlation function because of lack of dense gauges in their study; as a result, the areapoint variance was overestimated.

Another procedure often applied in the least squares curve-fitting problem, such as estimating parameters $r_{0}$ and $d_{0}$ in Eq. (9), is the Levenberg-Marquaedt algorithm (LMA; Press et al. 1988). The LMA is an iterative technique that optimizes the 
parameters so that the sum of the squares of the residual errors becomes minimal. Habib and Krajewski (2002) used the LMA to obtain function parameters with reasonable results. In some cases, however, the LMA is very sensitive to initial guess of the parameters, and converges only if the initial guess is already somewhat close to the final solution. In our case, the LMA fails to converge in fitting Eq. (9) although numerous initial guesses were tried.

The procedure applied in this study is the linear least squares method. The curvefitting problem in Eq. (9) can be linearized if we take a logarithm of both sides of Eq. (9). Thus, the parameters $r_{0}$ and $d_{0}$ in Eq. (9) can be estimated by the linear least squares method from all 465 pairs of $r$ and $d$ among $31 \mathrm{KSC}$ gauges for each time scale. The fitted function is presented in the inserted text in each panel of Fig. 6. A general feature of the correlation function shown in Fig. 6 is that the correlation and correlation distance increase with the integration time scale. At 1 -day scale, the parameter $d_{0}$ reaches a rather large value of $65.69 \mathrm{~km}$, thus the exponential part in Eq. (9) is close to 1, and the correlation structure is close to linear. The parameter $r_{0}$ represents correlation coefficient at zero-separation distance. The value of $r_{0}$ is less than 1 in all panels of Fig. 6 , which could be attributed to the small-scale rainfall variability and gauge measurement errors. When the integration time scale increases to $30 \mathrm{~min}$ or longer, where gauge-sampling errors are negligible (Wang et al 2008; Habib et al. 2001b), $r_{0}$ reaches a value of 0.9240.997 , which represents the natural variability at very small scales. At the separation distance of $2 \mathrm{~km}$, the correlation coefficient estimated from Eq. (9) is 0.588, 0.648, 0.700, $0.811,0.899$ and 0.950 for the time scales of $5,10,15,30,60$ min and 1 day, respectively. This coefficient decreases to $0.083,0.095,0.109,0.131,0.213$ and 0.620 as 
the separation distance increases to $30 \mathrm{~km}$. The rapid drop of the correlation level at relatively short distance is consistent with the fact that the short-lived and localized rainfall systems often occur in the KSC area.

\section{c. EVSM implementation}

The estimation of parameters $r_{0}$ and $d_{0}$ in Eq. (9) is crucial in deriving the correlation structure at small scales. The area-point variance in Eq. (8) can be estimated by using the derived correlation structure. In order to do so, the gauge separation distance in (9) needs to be expressed as

$$
d=\sqrt{\left(x_{1}-x_{2}\right)^{2}+\left(y_{1}-y_{2}\right)^{2}}
$$

in the Cartesian coordinate system, where $\left(x_{1}, y_{1}\right)$ and $\left(x_{2}, y_{2}\right)$ are two gauge positions. The distance between any two given points can be similarly expressed. Applying this expression into (9) and then substituting (9) into (8), (8) can be analytically integrated, and thus $\operatorname{var}\left(R_{g}-R_{t}\right)$ can be computed. Clearly, the area-point variance $\operatorname{var}\left(R_{g}-R_{t}\right)$ is only dependent on the relative gauge location inside the radar grid.

As discussed earlier, the variance of radar-gauge difference $\operatorname{var}\left(R_{r}-R_{g}\right)$ can be estimated using (6). Once $\operatorname{var}\left(R_{r}-R_{g}\right)$ and $\operatorname{var}\left(R_{g}-R_{t}\right)$ are obtained, the radar error variance $\operatorname{var}\left(R_{r}-R_{t}\right)$ can be easily determined as the difference between these two variances according to (5). Thus, the entire EVSM implementation is completed.

\section{d. EVSM results}

Figure 7 displays the results of various statistics used in the error separation 
analysis in the form of boxplots at time scales from 5-min to 1-day. The boxplot presents a quick sketch of the distribution of the underlying data at 31 gauges. The inter-quartile range extending from the lower quartile (25th) to the upper quartile (75th) is plotted as the black box, and the median is denoted as white bar inside the box in Fig. 7. The vertical line ("whisker") extends from both ends of the box to the maximum and minimum. Figures 7a-c represent the normalized standard errors of radar, gauge rain rates and radar-gauge rain-rate differences with respect to the true rain rates. These normalized standard errors are defined as the square roots of the variances from Eqs. (5), (8) and (6), divided by the gauge mean rain rate, respectively. The normalization makes the standard errors with different magnitudes dimensionless, and thus comparisons among different time scales meaningful. Standard deviations of gauge and radar rain rates are also normalized by the same gauge mean. Normalized standard deviations of radar (Fig. 7d) and gauge (Fig. 7e) rain rates do not greatly fluctuate with time scales. However, all 3 normalized standard errors regularly drop with the time scale, and the gauge error (Fig.7b) filters out faster than the radar error (Fig. 7a) via time averaging. The standard error of radar-gauge rain rate differences (Fig. 7c) is significantly greater than the radar error (Fig. 7a). While the standard error of radar-gauge rainfall differences (Fig. 7c) decreases with the time scale, the correlation coefficient (Fig. 7f) between radar and gauge rain rates increases. At 1-day scale, the median correlation coefficient reaches about 0.93 . The correlation at this level seems high enough so that the difference between radar and gauge rain rates should be fairly small. However, Fig. 7c shows that the normalized standard error of radar-gauge rain-rate differences is still as high as $71 \%$ at the 1-day scale. This demonstrates that the high correlation does not guarantee the minor 
difference between two random variables such as rain rates from the radar and gauge, and only considering the correlation can be misleading sometimes.

One observation that can be extracted from Fig.7a is that the median error of radar rain rate estimation is about $100 \%$ at 5 -min scale and it drops to about $75 \%$ at the scales of 15-60 min. Even at 1-day scale, the error stays at level of about $60 \%$. This exhibits that large improvements of the radar-rain product are still possible, which could be done by rigorous data quality control, algorithm refinement and utilization of other concurrent observations such as rain measurements from disdrometers, vertical profilers, etc.

In order to better understand the error variances separated by the EVSM, we present Fig. 8 in the form of ratios of error variances. Figures $8 \mathrm{a}$ depicts the ratio of radar error variance (Eq. (5)) with respect to the overall radar-gauge difference variance (Eq. (6)) as function of the time scale. The ratio of the gauge error variance (Eq. (8)) to radargauge difference variance (Eq. (6)) is displayed in Fig. 8b. Figure 8c is for the ratio of the radar to gauge error variance. The upper whiskers are truncated at 60-min and 1-day scales in Fig. 8c. The extreme whiskers are due to small error variances at several gauges, and are not considered to be representative. At short time scales of 5-30 min, the gauge representativeness error, expressed as the median variance ratio, contributes about 40$50 \%$ to the radar-gauge differences (Fig. 8 b). When the time scale increases beyond 60 min, the gauge error (Fig. 7b) decreases much faster than the radar errors (Fig. 7a) so that the radar error reaches 3 times as large as the gauge error (Fig. 8c). Notice that the ratio in Fig. $8 \mathrm{~b}$ remains at approximately same level of $25 \%$ from 60 -min to 1-day scales, which suggests that the area-point error still contributes an important part of the radargauge differences. This may imply that the time averaging cannot effectively remove 
small-scale rain field variability.

A variety of previous relevant studies have been carried out to quantify the contribution of the gauge representativeness error to the total radar-gauge differences. Kitchen and Blackall (1992) estimated that gauge representativeness error were about 50$80 \%$ for instantaneous and hourly rainfall at the grid size of $3 \mathrm{~km} \times 3 \mathrm{~km}$. Anagnostou and Krajewski (1999) showed that the gauge rainfall uncertainty contributed up to $60 \%$ of the variance observed in radar-gauge differences for hourly rainfall at $2-4 \mathrm{~km}$ grid resolution. However, those two analyses were conducted after logarithmic transformations of ratios of radar-gauge pairs, and then the uncertainty levels equivalent to results without logarithmic transformation were approximately estimated. Thus, caution must be taken in when comparing their results with the results of this study.

Ciach and Krajewski (1999) reported the ratio of gauge error variance to the radar-gauge difference variance was in the order of $55-70 \%$ at $5-30 \mathrm{~min}$ scales. This ratio is relatively larger than our result (40-50\%). As discussed in section $4 \mathrm{~b}$, their ratio was overestimated, which resulted from the over-simplified correlation function because of the lack of gauge measurements at the scale of radar grid in their study. When the time scale increases to 1-day, the ratio of gauge error variance remains about $25 \%$ for both studies. At this scale, the correlation distance $d_{0}$ in Eq. (9) is as large as $65.7 \mathrm{~km}$ (Fig. 6), thus the correlation structure is dominated by the correlation jump $r_{0}$. The approximation in Ciach and Krajewski (1999) could be applicable at 1-day scale in our study, and both results are consequently consistent. 


\section{Concluding remarks}

TRMM GV radar rain rates are spatiotemporally matched and inter-compared with the quality-controlled gauge rain rates over the KSC area for the period of 2008 . It is found that the overall bias of radar rain estimates relative to gauge measurements over the 1-year period is about $-5.48 \%$. However, significant differences of concurrent radargauge rain rates exist at various time scales from $5 \mathrm{~min}$ to 1 day, which is mainly due to different spatial and temporal samplings between radar and gauge observations. The KMLB radar instantaneously samples rainfall at scan intervals of about 5 minutes with range resolutions of $0.25 \mathrm{~km}$ and variable vertical resolutions. The $\mathrm{KSC}$ gauges measure amounts of accumulative rainwater falling into their near-point orifices in increments of $0.254 \mathrm{~mm}$. Rain rate can often change by a factor of 10 over distances of $2 \mathrm{~km}$ or during time intervals of several minutes (Joss and Waldvogel 1990). Therefore, the gauge measurements cannot be representative of true rain rates in the entire area beneath the radar-sampled volume. Similarly, instantaneous radar observations in any given sampling volume may not be representative of rain intensities during the intervals between volumescans. Additionally, rain gauges may suffer systematic, mechanical and electrical problems (Wang et al. 2008), whereas the radar can be subjected to hardware calibration and stability issues, nonuniform beam-filling and attenuation effects (Wang and Wolff 2009). Moreover, algorithm-caused rain estimation biases could be introduced by the cubic spline method employed in the gauge rain interpolation and the $Z_{\mathrm{e}}-\mathrm{R}$ relationships used in the radar rain estimation. All these factors contribute to differences in the comparisons of radar-gauge rain rates. 
After the overall bias is removed, the radar-gauge difference variance is separated into the gauge area-point error variance and radar-rain estimation error variance. The area-point error in rain gauge rainfall, expressed as median variance ratio, contributes about $40-50 \%$ of the variance in radar-gauge differences at $5-30$ min scales, and remains about $25 \%$ at 1 -day scale. The adequately dense arrangement of rain gauges under the entire radar umbrella would be expected to improve gauge area representativeness. There is also plenty of room for improvement of the radar-rain estimation by rigorous data quality control, algorithm refinement and utilization of other concurrent information such as rain measurements from disdrometers, vertical profilers, etc.

This study is conducted for providing the uncertainty evaluation of radar rain-rate estimates as a TRMM GV effort. The results are helpful to better utilize GV radar-rain products to validate versatile spaced-based rain estimates from TRMM, as well as the proposed Global Precipitation Measurement, and other satellites. 


\section{Acknowledgments}

This study was funded by NASA Grant NNG07EJ50C. The authors would like to thank Dr. Ramesh Kakar (NASA Headquarters), Dr. Scott Braun (TRMM Project Scientist), Dr. Arthur Hou (GPM Project Scientist), and Mr. Richard Lawrence (Chief, TRMM Satellite Validation Office) for their support of this effort. We would also like to thank the TRMM GV colleagues D. A. Marks, D. S. Silberstein and J. L. Pippitt for providing TSP 2A-53 and KMLB radar gap information. Thanks also go to support staff at Kennedy Space Center for their routine operation of the rain gauge network. 


\section{References}

Adler R. F., G. J. Huffman, D. T. Bolvin, S. C. Urtis, and E. J. Nelkin, 2000: Tropical rainfall distributions determined using TRMM combined with other satellite and rain gauge information. J. Appl. Meteor., 39, 2007-2023.

Amitai, E., 2000: Systematic variation of observed radar reflectivity-rainfall rate relations in the Tropics. J. Appl. Meteor., 39, 2198-2208.

Anagnostou E. N., W. F. Krajewski, and J. A. Smith, 1999: Uncertainty quantification of mean-areal radar-rainfall estimates. J. Atmos. Oceanic Technol., 16, 206-215.

Barnston, A. G., 1991: An empirical method of estimating raingauge and radar rainfall measurement bias and resolution. J. Appl. Meteor., 30, 282-296.

Ciach J. G., and W. F. Krajewski, 1999: On the estimation of radar rainfall error variance. Adv. Water Resour., 22, 585-595.

$\longrightarrow$, and 2006: Analysis and modeling of spatial correlation structure in small-scale rainfall in Central Oklahoma. Adv. Water Resour., 29, 1450-1463.

Cressie, N.A.C., 1993: Statistics for Spatial Data. Wiley Series in Probability and Mathematical Statistics. John Wiley \& Sons, New York, 900 pp. 
Datta S., W. L. Jones, B. Roy, and A. Tokay, 2003: Spatial variability of surface rainfall as observed from TRMM field campaign data. J. Appl. Meteor., 42, 598-610.

Fisher B. L., 2007: Statistical error decomposition of regional-scale climatological precipitation estimates from the Tropical Rainfall Measuring Mission (TRMM). $J$. Appl. Meteor. Climatol., 46, 791-813.

Habib E., W. F. Krajewski, and G. J. Ciach, 2001a: Estimation of rainfall interstation correlation. J. Hydrometeor., 2, 621-629.

$\longrightarrow$, and A. Kruger, 2001b: Sampling errors of tipping-bucket rain gauge measurements. J. Hydrol. Eng., 6, 159-166.

$\longrightarrow$, and $\longrightarrow$ 2002: Uncertainty Analysis of the TRMM Ground-Validation Radar-Rain products: Application to the TEFLUN-B Field Campaign. J. Appl. Meteor., 41, 558-572.

, G. J. Ciach, and W. F. Krajewski, 2004: A method for filtering out raingauge representativeness errors from the verification distributions of radar and raingauge rainfall. Adv Water Resour., 27,967-80.

Houze, R. A., Jr., S. Brodzik, C. Schumacher, S. E. Yuter, 2004: Uncertainties in Oceanic Radar Rain Maps at kwajalein and implications for satellite validation. J. Climate 
Appl. Meteor., 43,1114-1132.

Joss, J., and A. Waldvogel, 1990: Precipitation measurement and hydrology. Radar in meteorology: Battan memorial and 40th anniversary radar meteorology conference, D. Atlas, Ed., Amer. Meteor. Soc., 577-606.

Kitchen M., and R. M. Blackall, 1992: Representativeness errors in comparisons between radar and gauge measurements of rainfall. J. Hydrol., 134, 13-33.

Kowalski, C. J., 1972: On the effect of non-normality on the distribution of the sample product-moment correlation coefficient. Appl. Stat., 27, 1-12.

Krajewski, W. F. and C. J. Duffy, 1988: Estimation of correlation structure for a homogeneous isotropic random field: a simulation study. Computers and Geosciences, 14, 113-122.

, G. J. Ciach, and E. Habib, 2003: An analysis of small-scale rainfall variability in different climatic regimes. Hydrol. Sci. J., 48, 151-62.

Kummerow C., W. Barnes, T. Kozu, J. Shiue, and J. Simpson, 1998: The Tropical Rainfall Measuring Mission (TRMM) sensor package. J. Atmos. Oceanic Technol., 15, 809-817. 
Lai, C. D., J. C. W. Rayner, and T. P. Hutchinson, 1999: Robustness of the sample correlation — The bivariate lognormal case. J. appl. Math. Decision Sci., 3, 7-19.

Press W. H., B. P. Flannery, S. A. Teukolsky, and W. T. Vetterling, 1988: Numerical Recipes: The Art of Scientific Computing, Cambridge University Press, 818 pp.

Shimizu, K., 1993: A bivariate mixed lognormal distribution with an analysis of rainfall data. J. Appl. Meteor., 32, 161-171.

Sieck, L. C., S. J. Burges, and M. Steiner, 2007: Challenges in obtaining reliable measurements of point rainfall, Water Resour. Res., 43, W01420, doi:10.1029/2005WR004519.

Simpson J. S., C. Kummerow, W.-K. Tao, and R. F. Adler, 1996: On the Tropical Rainfall Measuring Mission (TRMM). Meteor. Atmos. Phys., 60, 19-36.

Stedinger, J. R., 1981: Estimating correlations in multivariate streamflow models. Water Resour. Res., 17, 200-208.

Villarini, G., P. V. Mandapaka, W. F. Krajewski, and R. J. Moore, 2008: Rainfall and sampling uncertainties: A rain gauge perspective, J. Geophys. Res., 113, D11102, doi:10.1029/2007JD009214. 
Wang, J., B. L. Fisher, and D. B. Wolff, 2008: Estimating rain rates from tipping-bucket rain gauge measurements. J. Atmos. Oceanic Technol., 25, 43-56.

, and D. B. Wolff, 2009: Comparisons of Reflectivities from the TRMM Precipitation Radar and Ground-based Radars, J. Atmos. Oceanic Technol., 26, 857-875.

Wolff, D.B., D.A. Marks, E. Amitai, D.S. Silberstein, B.L. Fisher, A. Tokay, J. Wang, and J.L. Pippitt, 2005: Ground Validation for the Tropical Rainfall Measuring Mission (TRMM). J. Atmos. Oceanic Technol., 22, 365-380.

Zawadzki I., 1975: On radar-raingage comparison. J. Appl. Meteor., 14, 1430-1436. 


\section{List of Figures}

FIG.1. Aerial map of KSC gauge network along with $2 \mathrm{~km} \times 2 \mathrm{~km}$ Cartesian grids of TSP 2A-53 radar-rain map. The dots denote locations of the individual gauges with their identification numbers indicated on the right. The grids not containing gauges are not used in the analysis. The KMLB radar is about $50 \mathrm{~km}$ south of the center of the KSC gauge network.

FIG. 2. Cumulative rainfall averaged from all gauges and radar grids over the gauges, respectively, during the 1-year period of 2008.

FIG. 3. Scatter plots of radar and gauge rain rates for time scales of 5-, 10-, 15-, 30-, 60min and 1-day. Both radar and gauge rain rates are plotted in the same logarithmic scale for all time scales. The correlation coefficient is shown at the top left of each panel.

FIG. 4. Frequency distributions of radar-gauge rain-rate differences for time scales of 5-, $10-, 15-, 30-, 60-m i n$ and 1-day. Each distribution is constructed using all rainy radargauge data pairs covering the 1-year period of 2008 and is binned at $0.5 \mathrm{~mm} \mathrm{~h}^{-1}$ intervals. The mean and standard deviation of radar-gauge rain-rate differences are shown in each panel.

FIG. 5. (a) Percentage of gauge zero-rainfall occurrences conditional on radar rainfall existence in the $2 \mathrm{~km} \mathrm{X} 2 \mathrm{~km}$ radar grid over the gauge. (b) Percentage of radar zero- 
rainfall occurrences in the $2 \mathrm{~km} \mathrm{X} 2 \mathrm{~km}$ radar grid conditional on rainfall existence at the gauge inside the radar grid. (c) Percentage of gauge missing-rainfall. (d) Percentage of radar missing-rainfall.

FIG. 6. Scatter plots of spatial correlation coefficients as function of gauge separation distances estimated from pairs of gauge rain accumulations at time scales of 5-, 10-, 15-, 30-, 60-min and 1-day. The exponential fits for the estimated coefficients are also presented (solid line).

FIG. 7. Boxplots of various statistics used in the error separation analysis for 31 gauges at $5,10,15,30,60-\min$ and 1-day scales. The inter-quartile range extending from the lower quartile (25th) to the upper quartile (75th) is plotted as the black box, and the median is denoted as white bar inside the box. The vertical line represents whiskers extend from both ends of the box to the maximum and minimum. (a) Normalized standard errors of radar rain rates. (b) Normalized standard errors of gauge rain rates. (c) Normalized standard errors of radar-gauge rain-rate differences. (d) Normalized standard deviations of radar rain rates. (e) Normalized standard deviations of gauge rain rates. (f) Correlation coefficients between radar and gauge rain rates. The normalized standard error or deviation is defined as the square root of the variance divided by the gauge mean rain rate.

FIG. 8. Boxplots of error variance ratios for 31 gauges at 5, 10, 15, 30, 60-min and 1-day scales. (a) The ratio of radar error variance to radar-gauge difference variance. (b) The 
ratio of gauge error variance to radar-gauge difference variance. (c) The ratio of radar error variance to gauge error variance. 
TABLE 1 . Monthly KMLB radar gap statistics and missing rain percentages due to the gaps in 2008. The missing rain percentages are estimated from the percentages of gauge rainfall during the radar gaps.

\begin{tabular}{|c|c|c|c|c|}
\hline Month & Number of gaps & $\begin{array}{c}\text { Gap length } \\
\text { (min) }\end{array}$ & $\begin{array}{c}\text { Gap percentage } \\
(\%)\end{array}$ & $\begin{array}{c}\text { Missing rain } \\
(\%)\end{array}$ \\
\hline Jan & 13 & 794 & 1.78 & 0.11 \\
\hline Feb & 8 & 208 & 0.50 & 0.00 \\
\hline Mar & 164 & 3504 & 7.85 & 1.51 \\
\hline Apr & 138 & 3289 & 7.61 & 0.36 \\
\hline May & 13 & 1401 & 3.14 & 0.05 \\
\hline Jun & 6 & 509 & 1.18 & 1.12 \\
\hline Jul & 27 & 7144 & 16.00 & 35.97 \\
\hline Aug & 12 & 537 & 1.20 & 1.24 \\
\hline Sep & 9 & 3683 & 8.53 & 0.76 \\
\hline Oct & 11 & 1030 & 2.31 & 0.02 \\
\hline Nov & 6 & 788 & 1.82 & 0.31 \\
\hline Dec & 9 & 405 & 0.91 & 0.00 \\
\hline Total & 416 & 23292 & 4.42 & 3.49 \\
\hline
\end{tabular}


TABLE 2. Monthly KMLB radar and KSC gauge rainfall, and the radar rainfall bias relative to gauge rainfall in 2008 .

\begin{tabular}{|c|c|c|c|}
\hline Month & Radar (mm) & Gauge $(\mathrm{mm})$ & Bias (\%) \\
\hline Jan & 108.2 & 135.9 & -20.33 \\
\hline Feb & 52.7 & 48.5 & 8.78 \\
\hline Mar & 30.9 & 31.0 & -0.51 \\
\hline Apr & 69.9 & 72.7 & -3.92 \\
\hline May & 20.3 & 29.4 & -30.92 \\
\hline Jun & 98.3 & 134.6 & -26.97 \\
\hline Jul & 58.0 & 58.9 & -1.60 \\
\hline Aug & 589.7 & 678.4 & -13.07 \\
\hline Sep & 204.2 & 162.7 & 25.45 \\
\hline Oct & 175.8 & 138.0 & 27.47 \\
\hline Nov & 53.2 & 54.3 & -2.10 \\
\hline Dec & 13.2 & 15.5 & -14.64 \\
\hline Total & 1474.4 & 1560.0 & -5.48 \\
\hline
\end{tabular}




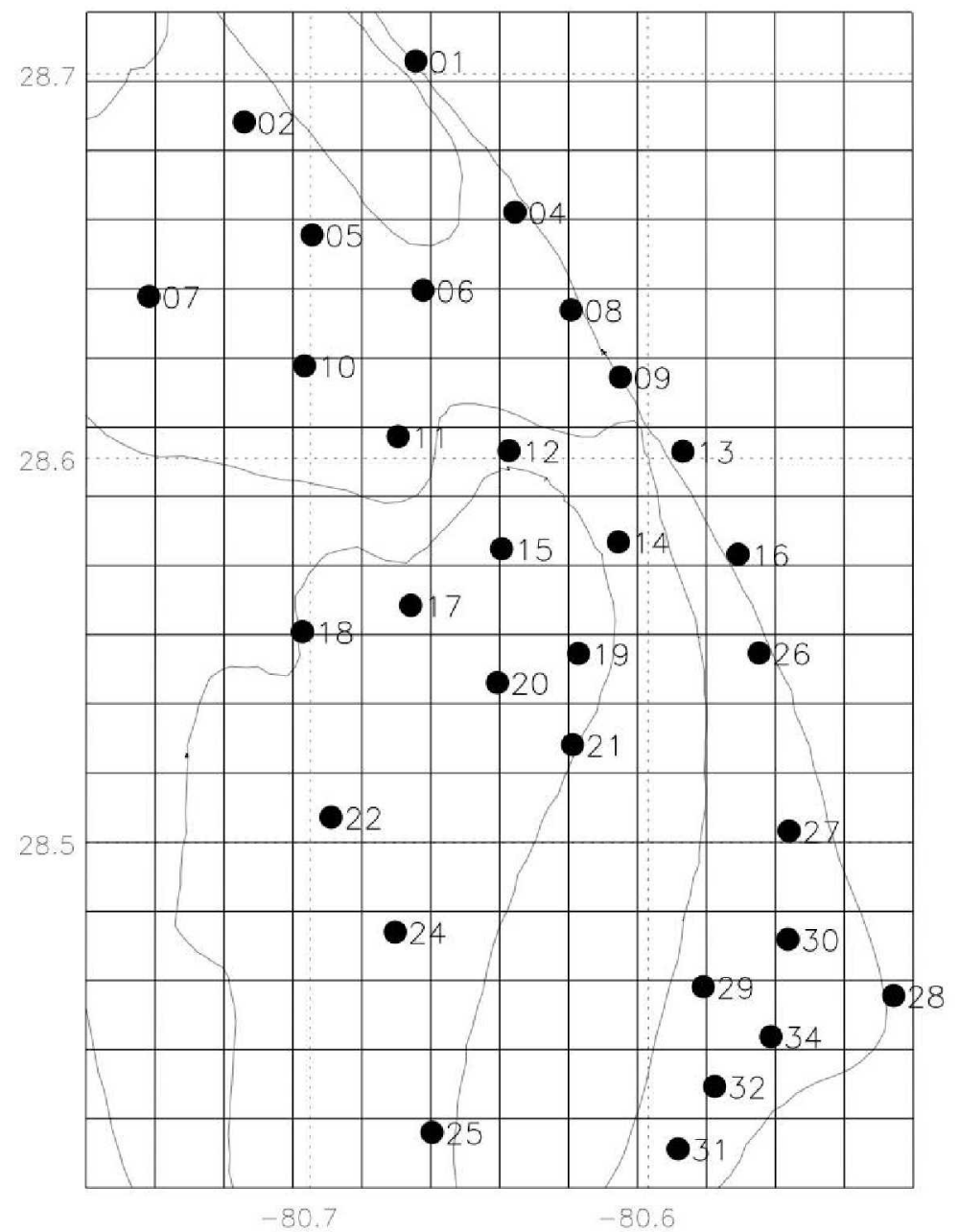

FIG.1. Aerial map of KSC gauge network along with $2 \mathrm{~km} \times 2 \mathrm{~km}$ Cartesian grids of TSP 2A-53 radar-rain map. The dots denote locations of the individual gauges with their identification numbers indicated on the right. The grids not containing gauges are not used in the analysis. The KMLB radar is about $50 \mathrm{~km}$ south of the center of the KSC gauge network. 


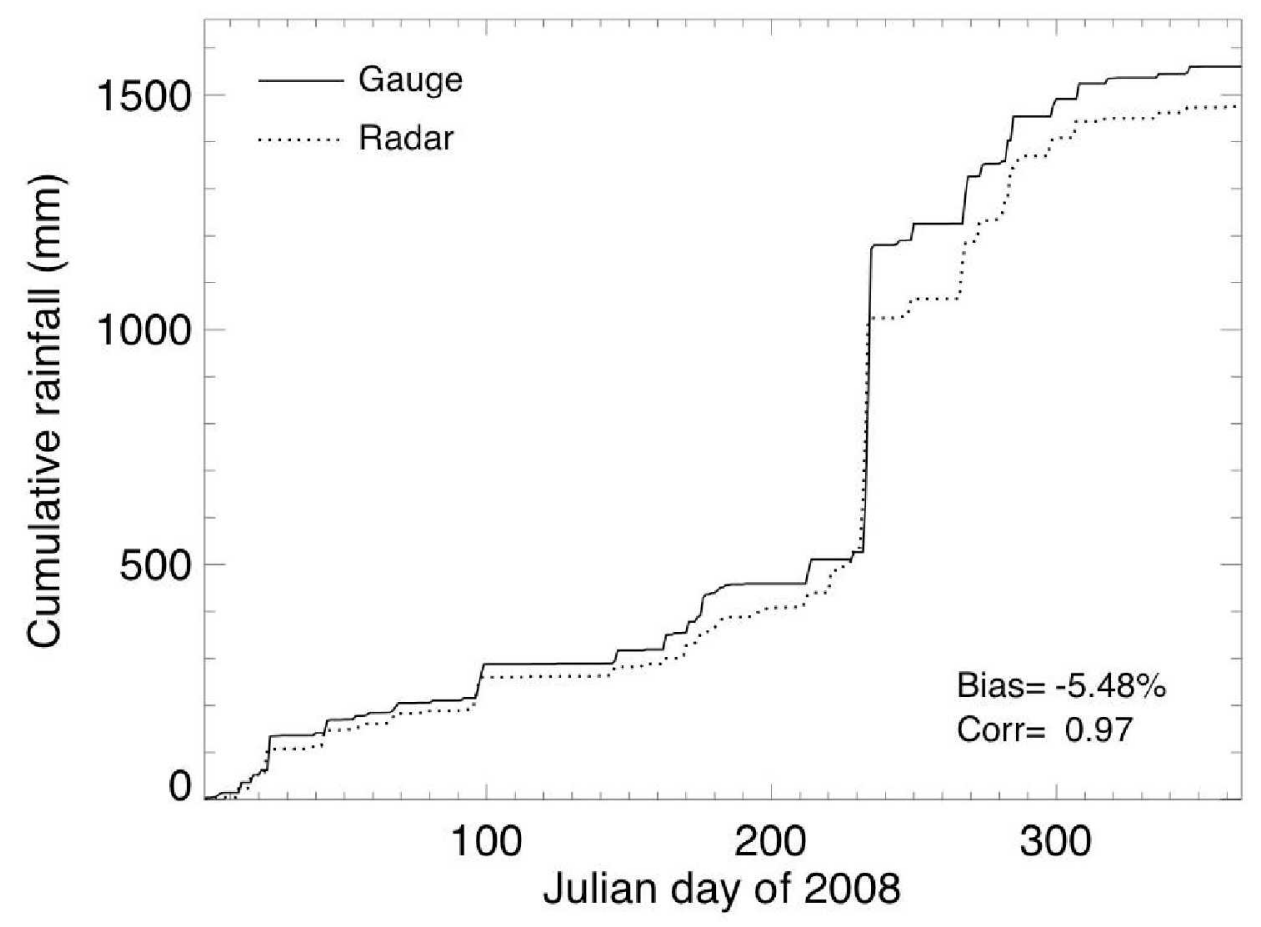

FIG. 2. Cumulative rainfall averaged from all gauges and radar grids over the gauges, respectively, during the 1 -year period of 2008. 

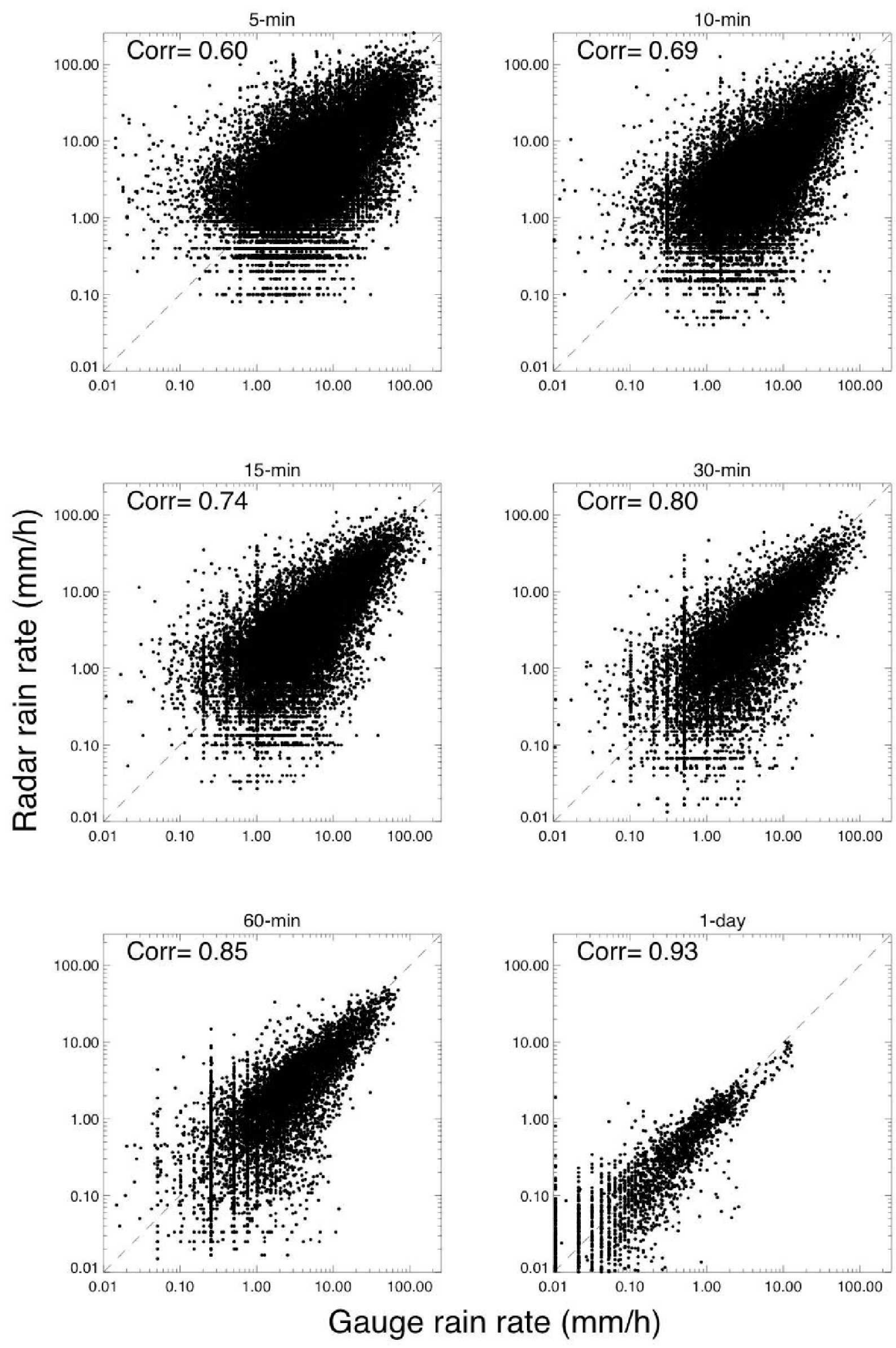

FIG. 3. Scatter plots of radar and gauge rain rates for time scales of 5-, 10-, 15-, 30-, 60min and 1-day. Both radar and gauge rain rates are plotted in the same logarithmic scale for all time scales. The correlation coefficient is shown at the top left of each panel. 

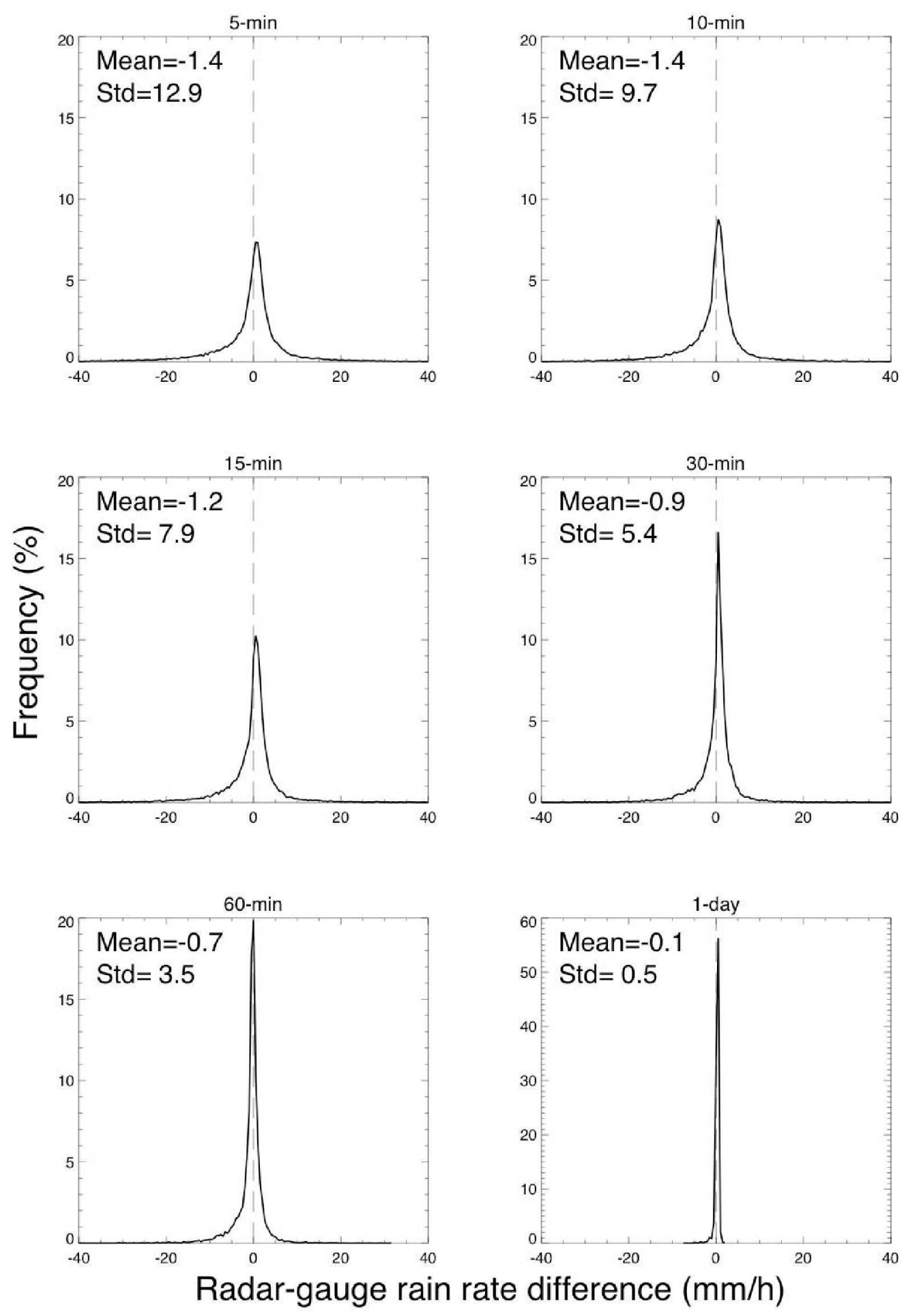

FIG. 4. Frequency distributions of radar-gauge rain-rate differences for time scales of 5-, $10-, 15-, 30-, 60-\mathrm{min}$ and 1-day. Each distribution is constructed using all rainy radargauge data pairs covering the 1-year period of 2008 and is binned at $0.5 \mathrm{~mm} \mathrm{~h}^{-1}$ intervals. The mean and standard deviation of radar-gauge rain-rate differences are shown in each panel. 

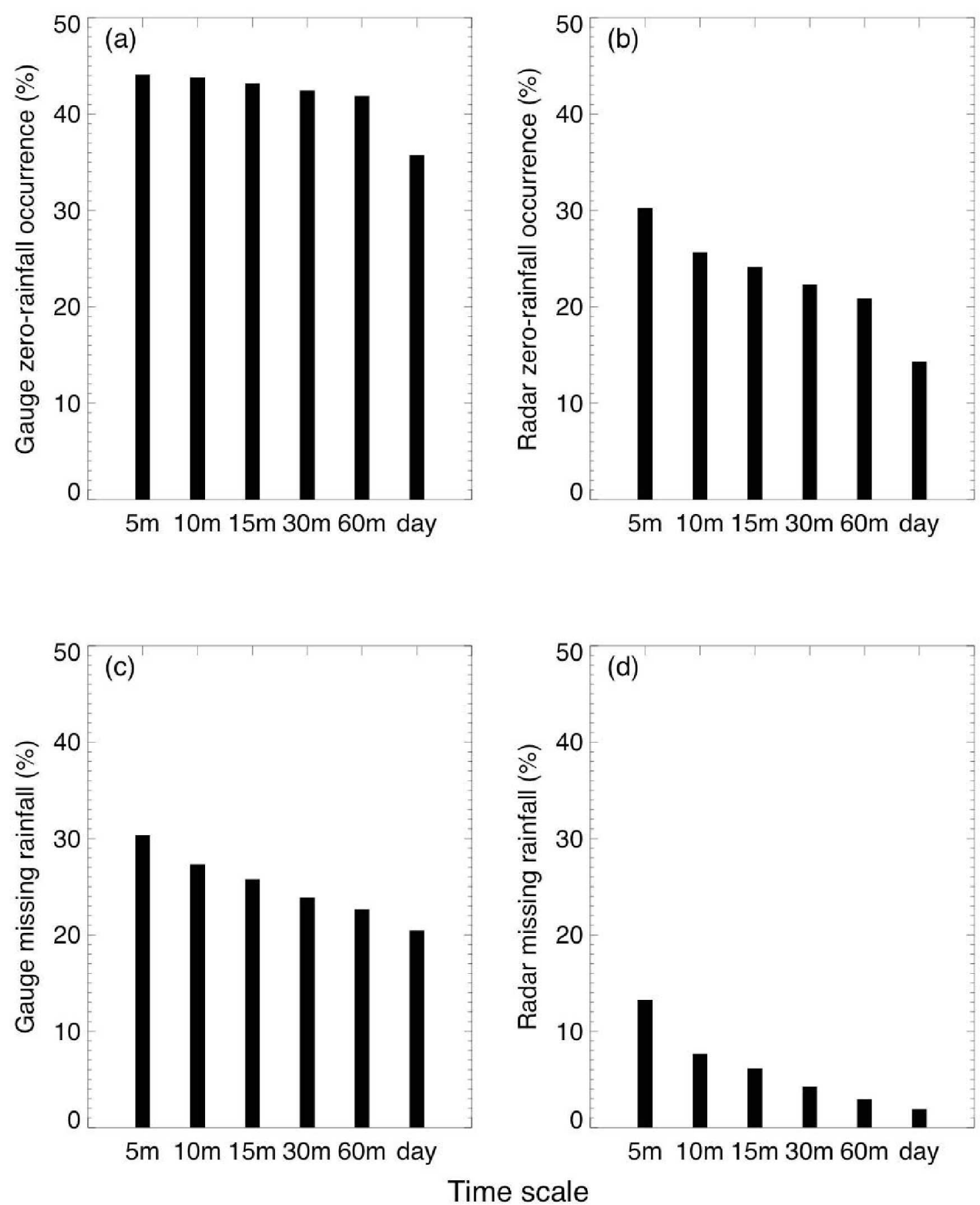

FIG. 5. (a) Percentage of gauge zero-rainfall occurrences conditional on radar rainfall existence in the $2 \mathrm{~km} \times 2 \mathrm{~km}$ radar grid over the gauge. (b) Percentage of radar zerorainfall occurrences in the $2 \mathrm{~km} \times 2 \mathrm{~km}$ radar grid conditional on rainfall existence at the gauge inside the radar grid. (c) Percentage of gauge missing-rainfall. (d) Percentage of radar missing-rainfall. 

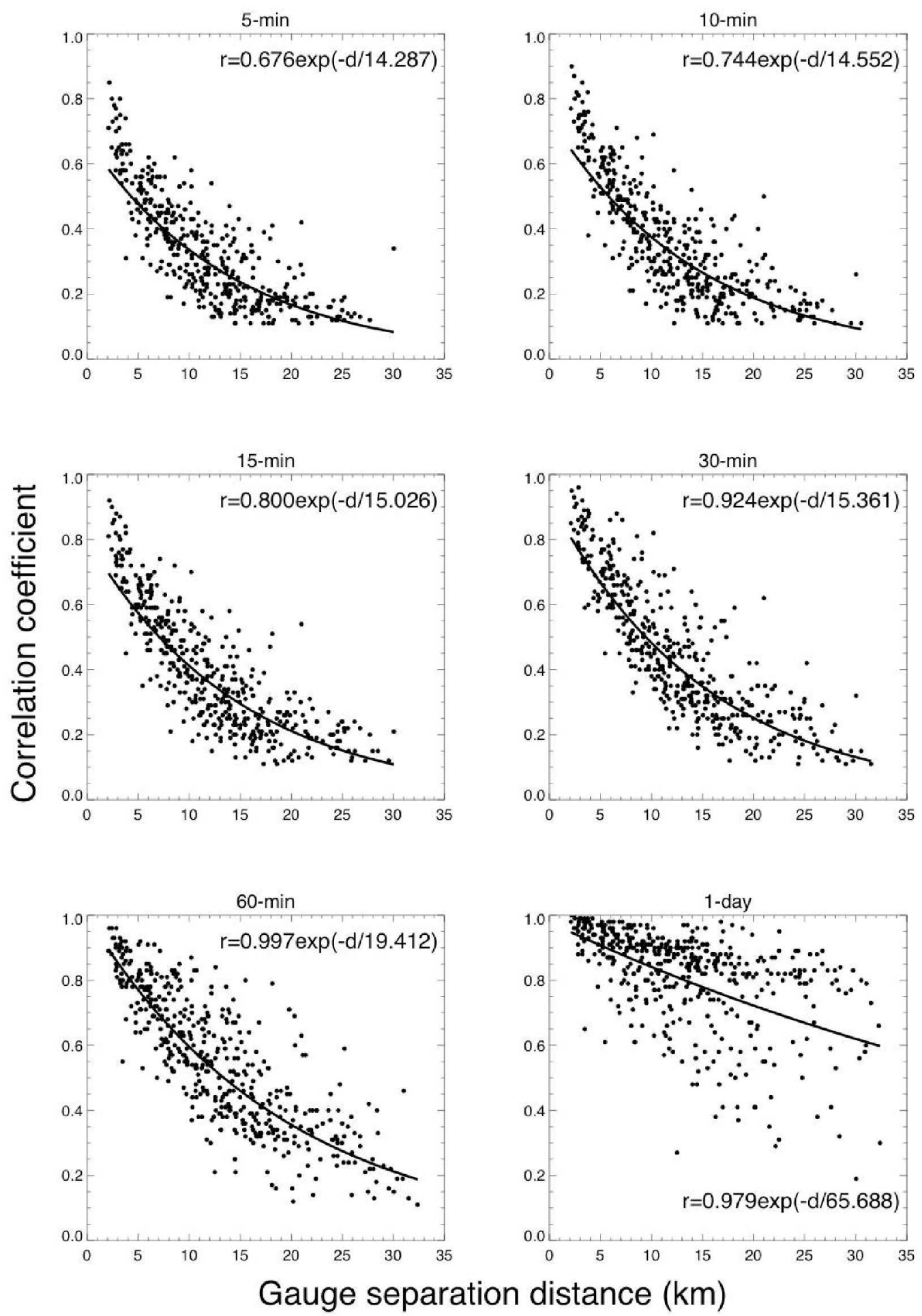

FIG. 6. Scatter plots of spatial correlation coefficients as function of gauge separation distances estimated from pairs of gauge rain accumulations at time scales of 5-, 10-, 15-, 30-, 60-min and 1-day. The exponential fits for the estimated coefficients are also presented (solid line). 

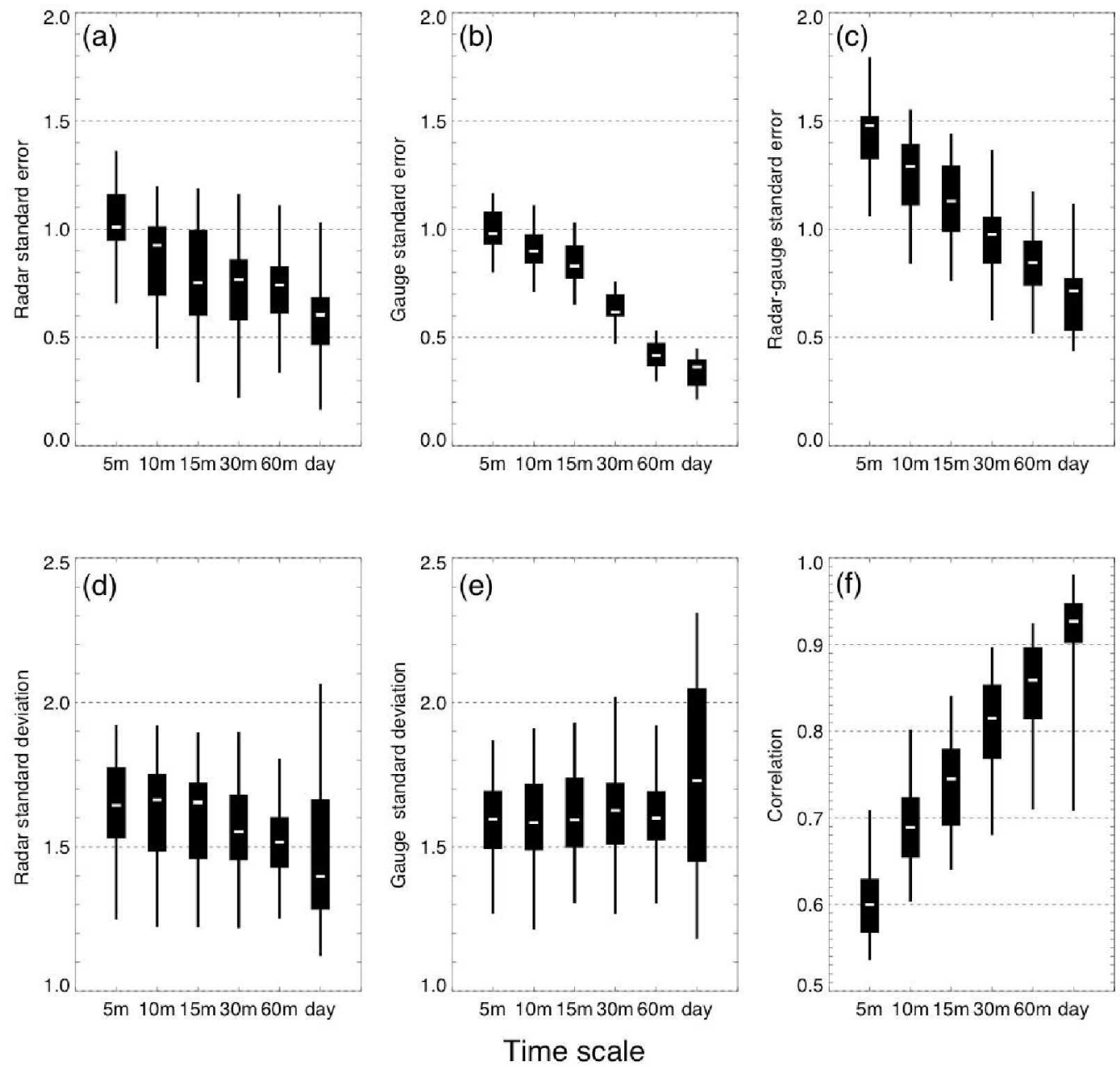

FIG. 7. Boxplots of various statistics used in the error separation analysis for 31 gauges at $5,10,15,30,60-\mathrm{min}$ and 1-day scales. The interquartile range extending from the lower quartile (25th) to the upper quartile (75th) is plotted as the black box, and the median is denoted as white bar inside the box. The vertical line represents whiskers extend from both ends of the box to the maximum and minimum. (a) Normalized standard errors of radar rain rates. (b) Normalized standard errors of gauge rain rates. (c) Normalized standard errors of radar-gauge rain-rate differences. (d) Normalized standard deviations of radar rain rates. (e) Normalized standard deviations of gauge rain rates. (f) Correlation coefficients between radar and gauge rain rates. The normalized standard error or deviation is defined as the square root of the variance divided by the gauge mean rain rate. 

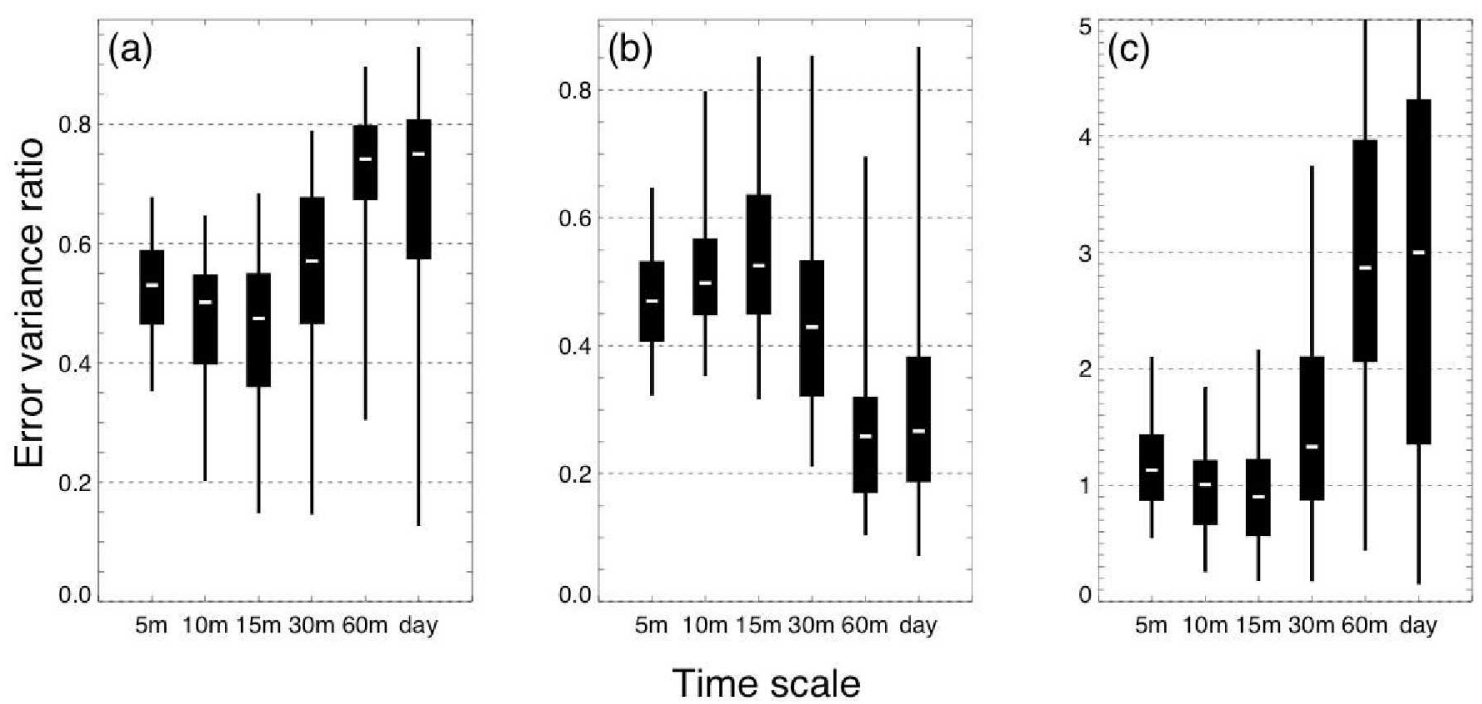

FIG. 8. Boxplots of error variance ratios for 31 gauges at 5, 10, 15, 30, 60-min and 1-day scales. (a) The ratio of radar error variance to radar-gauge difference variance. (b) The ratio of gauge error variance to radar-gauge difference variance. (c) The ratio of radar error variance to gauge error variance. 


\title{
Evaluation of TRMM Ground-Validation Radar-Rain Errors Using Rain Gauge Measurements
}

\author{
Jianxin Wang (Jianxin.Wang@nasa.gov) \\ David B. Wolff (David.B.Wolff@nasa.gov) \\ Submitted to Journal of Applied Meteorology and Climatology
}

\section{Popular Summary}

The Tropical Rainfall Measuring Mission (TRMM) is a satellite-based program to estimate global tropical rainfall. A wealth of data has been obtained since the launch of the TRMM satellite in November 1997. The accuracy of the satellite-based rain products, however, is affected by discrete temporal sampling and remote spaceborne rain retrieval algorithms. The TRMM ground validation (GV) program was established to verify the satellite rain products, which can be carried out by comparing the TRMM products with ground observations from ground sensors, such as rain gauges, radars and disdrometers. The GV radar-rain products play a crucial role in this validation effort. TRMM Standard Product 2A-53, an instantaneous GV radar rain-rate map, is often used as a main tool for the validation analysis. However, the radar rain-rate map has its own quality issues that must be quantified before it can be used to properly validate the satellite rain estimates. The major obstacle in evaluation of the quality of the radar rain-rate map is the lack of "ground truth" reference at the radar pixel scale $(2 \mathrm{~km} \times 2 \mathrm{~km})$. The surface rainfall measurements from rain gauges are customarily approximated as the "ground truth" to 
assess and calibrate radar-rain estimates. The gauge gives the surface rainfall amounts in a near-point area as rainwater impinges on its $30.5-\mathrm{cm}$ (or so) diameter collection orifice. The radar, in contrast, does not directly measure surface rainfall amounts, but measures reflectivity factors aloft at its resolution cell in the order of 1 to $10 \mathrm{~km}^{2}$. The areaaveraged surface rainfall is subsequently estimated via converting raw reflectivity using any number of reflectivity - rain-rate $\left(\mathrm{Z}_{\mathrm{e}}-\mathrm{R}\right)$ relationships. Even if both gauge near-point measurements and radar area-averaged rainfall estimates are assumed to be accurate, the large resolution difference of 7-8 orders of magnitude still obviously causes problematic comparisons between two sensors. Because gauges lack areal representativeness, the gauge rain measurements cannot be directly treated as the "ground truth" reference for the area-averaged rainfall. Therefore, the large radar-gauge discrepancies cannot be treated as radar-rain estimation error.

This study uses quality-controlled gauge data to compare with TRMM GV radar rain rates in an effort to provide quantitative evaluation of the GV radar-rain product error characteristics. The error variance separation method is adapted to partition the radar-gauge difference variance into the radar-rain estimation error variance and the gauge representativeness error variance. The results provide relatively reliable quantitative uncertainty evaluation of TRMM GV radar rain estimates at various times scales ranging from $5 \mathrm{~min}$ to 1 day, and are helpful to better understand the differences between measured radar and gauge rain rates. It is envisaged that this study will contribute to better utilization of GV radar rain products to validate versatile spacedbased rain estimates from TRMM, as well as the proposed Global Precipitation Measurement, and other satellites. 